\title{
Trial-by-trial adjustments of cognitive control following errors and response conflict are altered in pediatric obsessive compulsive disorder
}

\author{
Yanni Liu ${ }^{1}{ }^{*}$, William J. Gehring ${ }^{2}$, Daniel H. Weissman ${ }^{2}$, Stephan F. Taylor ${ }^{1}$ and Kate Dimond Fitzgerald ${ }^{1}$ * \\ ${ }^{1}$ Department of Psychiatry, Medical School, University of Michigan, Ann Arbor, MI, USA \\ 2 Department of Psychology, University of Michigan, Ann Arbor, MI, USA
}

Edited by:

Michael H. Bloch, Yale University, USA

Reviewed by:

Ewgeni Jakubovski, University of Heidelberg, Germany

Emily R. Stern, Mount Sinai School of Medicine, USA

\section{${ }^{*}$ Correspondence:}

Yanni Liu and Kate Dimond Fitzgerald, Department of Psychiatry, University of Michigan, 4250 Plymouth Road,

Ann Arbor, MI 48109, USA.

e-mail: yanniliu@umich.edu;

krd@umich.edu

\begin{abstract}
Background: Impairments of cognitive control have been theorized to drive the repetitive thoughts and behaviors of obsessive compulsive disorder (OCD) from early in the course of illness. However, it remains unclear whether altered trial-by-trial adjustments of cognitive control characterize young patients. To test this hypothesis, we determined whether trial-by-trial adjustments of cognitive control are altered in children with OCD, relative to healthy controls. Methods: Forty-eight patients with pediatric OCD and 48 healthy youth performed the Multi-Source Interference Task. Two types of trial-by-trial adjustments of cognitive control were examined: post-error slowing (i.e., slower responses after errors than after correct trials) and post-conflict adaptation (i.e., faster responses in high-conflict incongruent trials that are preceded by other high-conflict incongruent trials, relative to lowconflict congruent trials). Results: While healthy youth exhibited both post-error slowing and post-conflict adaptation, patients with pediatric OCD failed to exhibit either of these effects. Further analyses revealed that patients with low symptom severity showed a reversal of the post-conflict adaptation effect, whereas patients with high symptom severity did not show any post-conflict adaptation. Conclusion: Two types of trial-by-trial adjustments of cognitive control are altered in pediatric OCD. These abnormalities may serve as early markers of the illness.
\end{abstract}

Keywords: cognitive control, post-conflict adaptation, error monitoring, interference, post-error slowing, pediatric obsessive compulsive disorder

\section{INTRODUCTION}

Patients with obsessive compulsive disorder (OCD) often have difficulty controlling intrusive thoughts (obsessions) and behaviors (compulsions) despite realizing that they do not "make sense" (American Psychiatric Association, 2000). One framework for interpreting these deficits posits that they arise from impaired $\operatorname{cog}$ nitive control: the ability to voluntarily control behavior, monitor the consequences of one's actions, and make behavioral adjustments when necessary (Norman and Shallice, 1986). Consistent with this view, OCD has been linked to disturbances of cognitive control in response inhibition (Rosenberg et al., 1997; Bannon et al., 2002; Aycicegi et al., 2003; Watkins et al., 2004; Chamberlain et al., 2006) and negative priming (Enright and Beech, 1993) paradigms.

Problems with making trial-by-trial adjustments of cognitive control may also be present in OCD, especially under conditions that are characterized by high levels of processing conflict. For example, an OCD patient may "get stuck" in a repetitive pattern of action like repeatedly washing her hands because she does not adjust appropriately to the conflict between (a) trying to perform a more productive task and (b) feeling compelled to wash her hands again. An appropriate adjustment might involve pausing to prevent an error (e.g., another hand-washing episode) or further increasing attention to the goal of performing the more productive task.

These two types of behavioral adjustments are often observed in laboratory studies of response-interference tasks (e.g., the Stroop task). The first type of adjustment is called post-error slowing. Posterror slowing is thought to reflect a greater emphasis on response accuracy (at the expense of response speed) following an error, which serves to prevent future errors (Botvinick et al., 2001). Operationally, it is defined as slower responses in correctly performed trials that are preceded by high-conflict error trials (errorCorrect) as compared to low-conflict correctly performed trials (correctCorrect; Rabbitt, 1966; Laming, 1968; Botvinick et al., 2001). The second type of adjustment is called post-conflict adaptation. Post-conflict adaptation is thought to reflect greater focusing of attention on relevant information following high-conflict, correctly performed incongruent trials (e.g., Botvinick et al., 2001; Egner and Hirsch, 2005). Operationally, it is defined as faster responses in incongruent trials that are preceded by other incongruent trials (iI trials) as compared to congruent trials (cI trials; e.g., Egner et al., 2008). Notably, both post-error slowing and post-conflict adaptation are "appropriate" reactions to high levels of processing conflict because they each serve to enhance future performance. 
Support for the view that OCD is linked to altered trial-by-trial adjustments of cognitive control comes from multiple sources. First, aberrant post-error slowing has been reported in adults with OCD symptoms (Fitzgerald et al., 2005), although such effects are not always observed (Hajcak and Simons, 2002; Endrass et al., 2008). Second, altered post-conflict adaptation has been observed in patients with adult OCD compared to controls (Meiran et al., 2011) and in college students with high (vs. low) levels of subclinical OCD symptoms (Soref et al., 2008). Given the typical onset of OCD during childhood and adolescence (Pauls et al., 1995), altered trial-by-trial adjustments of control (i.e., post-error slowing or post-conflict adaptation) may also be present in pediatric OCD and may predict the severity of symptoms experienced by individual patients. To our knowledge, however, no prior study has explored these possibilities.

To investigate whether trial-by-trial adjustments of performance are altered in pediatric OCD, we asked patients with pediatric OCD and matched controls to perform the Multi-Source Interference Task (MSIT; Bush and Shin, 2006). The MSIT is designed to evoke high levels of processing conflict while remaining easy enough for children to perform (Bush and Shin, 2006; Fitzgerald et al., 2010) and thus appears well-suited for exploring altered cognitive control in pediatric OCD.

We made two predictions. First, based on previous studies of healthy youth (Davies et al., 2004; Santesso et al., 2006; Ladouceur et al., 2007), we hypothesized that healthy youth would exhibit both post-error slowing and post-conflict adaptation. Second, based on previous studies showing impaired trial-by-trial adjustments of performance in adults with OCD (Fitzgerald et al., 2005; Soref et al., 2008; Meiran et al., 2011), we predicted that patients with pediatric OCD would exhibit alterations of both (a) post-error slowing and (b) post-conflict adaptation.

\section{MATERIALS AND METHODS PARTICIPANTS}

The participants (age range: $8-19$ years) were 48 patients with pediatric OCD and 48 age- and gender-matched healthy youth
(Table 1). All participants were evaluated using the KiddieSchedule for Affective Disorders-Present and Lifetime Version (Kaufman et al., 1997), the Multidimensional Anxiety Scale for Children (MASC, March et al., 1997), and the Child Depression Inventory (CDI, Kovacs, 1992). Patients were also evaluated using the Children's Yale-Brown Obsessive Compulsive Scale (CYBOCS, Goodman et al., 1989). Socio-economic status (SES) was evaluated using the Hollingshead-Redlich index (Hollingshead and Redlich, 1958). Among patients, comorbid diagnoses were separation anxiety disorder $(n=4)$, generalized anxiety disorder $(n=2)$, anxiety disorder not otherwise specified (NOS, $n=2$ ), depressive disorder NOS $(n=5)$, major depressive disorder $(n=2)$, and tics $(n=6)$. Patients with attention deficit hyperactivity disorder or autism spectrum illness were excluded. Twenty-four patients were taking medications at the time of the study, including fluoxetine $(n=14)$, sertraline $(n=6)$, fluvoxamine $(n=1)$, citalopram $(n=2)$, and temazepam $(n=1)$. After providing each participant and his/her parents with a complete description of the study, written informed assent and consent, respectively, were obtained from pediatric participants and their parents.

\section{EXPERIMENTAL PARADIGM}

In each trial of the MSIT (Figure 1), participants were required to report the identity of the unique digit among three digits -1 , 2 , or 3 - by pressing a key on a keypad. The digits 1,2 , and 3, respectively, were mapped to the index, middle, and ring finger of the right hand. In incongruent trials (e.g. " 322 ”), the unique digit (e.g., " 3 ") appeared in a spatial position (e.g., left) that engendered a conflicting response (e.g., index finger), relative to the correct response (e.g., ring finger). The identity of the two identical distracter digits (e.g., "2") also engendered a conflicting response (e.g., middle finger), relative to the correct response (e.g., ring finger). In congruent trials (e.g., "020”), the unique digit (e.g., "2") appeared in a spatial position (e.g., middle) that engendered a non-conflicting response (e.g., middle finger), relative to the correct response (e.g., middle finger). The two identical distracter digits were always zeros and therefore also did not engender a

Table 1 | Demographic Information for the OCD and Control Groups.

\begin{tabular}{|c|c|c|c|c|c|c|}
\hline & \multicolumn{3}{|c|}{ All participants } & \multicolumn{3}{|c|}{$\begin{array}{l}\text { Participants included in the post-error } \\
\text { slowing analysis (incongruent) }\end{array}$} \\
\hline & OCD & Control & Difference & OCD & Control & Difference \\
\hline Age (mean $\pm S D)$ & $14.0 \pm 3.1$ & $13.9 \pm 3.1$ & ns & $14.8 \pm 2.9$ & $14.8 \pm 2.9$ & ns \\
\hline Gender (female: male) & $26: 22$ & $26: 22$ & ns & $18: 10$ & $11: 14$ & ns \\
\hline SES (mean \pm SD) & $2.1 \pm 0.4$ & $2.2 \pm 0.4$ & ns & $2.1 \pm 0.3$ & $2.3 \pm 0.4$ & ns \\
\hline Education (mean $\pm \mathrm{SD}$ ) & $8.9 \pm 3.0$ & $8.8 \pm 3.0$ & ns & $10.1 \pm 2.9$ & $9.5 \pm 2.4$ & ns \\
\hline MASC (mean \pm SD) & $49.2 \pm 16.8$ & $27.9 \pm 14.3$ & * & $49.0 \pm 18.5$ & $29.8 \pm 11.1$ & $*$ \\
\hline $\mathrm{CDI}($ mean $\pm \mathrm{SD})$ & $9.5 \pm 6.7$ & $2.7 \pm 3.4$ & * & $10.5 \pm 6.7$ & $3.5 \pm 4.0$ & * \\
\hline Current CYBOCs (mean \pm SD) & $18.1 \pm 7.5$ & & & $18.3 \pm 7.7$ & & \\
\hline Medicated: unmedicated & $23: 25$ & & & $11: 14$ & & \\
\hline
\end{tabular}

SES, socio-economic status, assessed by Hollingshead-Redlich Scale; CDI, Children's Depression Inventory; CYBOCS, Children's Yale-Brown Obsessive Compulsive

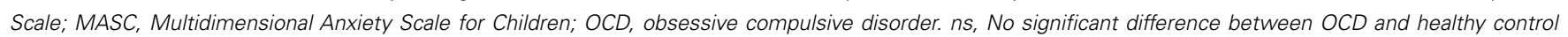
$(p>0.05) ;{ }^{*}$ significant difference at $p<0.05$. 


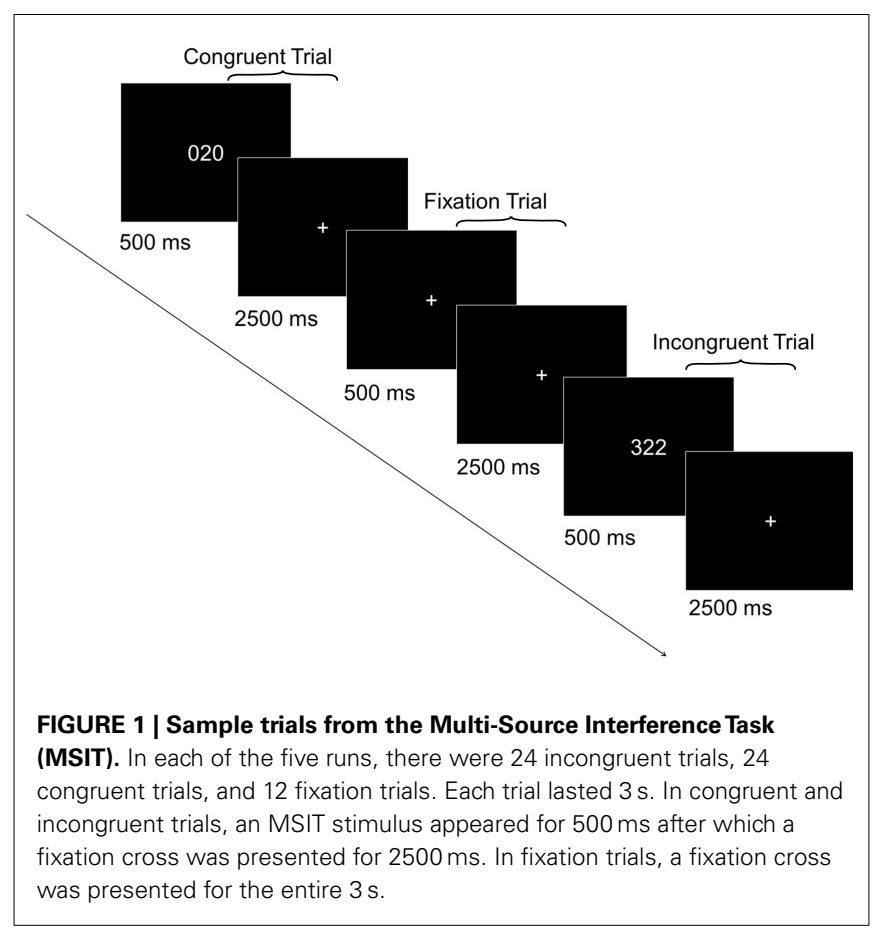

conflicting response, relative to the correct response (e.g., middle finger).

All participants performed five runs of the MSIT. Each run consisted of 24 incongruent trials, 24 congruent trials, and 12 fixation trials presented in a random order. In each congruent and incongruent trial, a three-digit stimulus appeared for $500 \mathrm{~ms}$, followed by a $2500-\mathrm{ms}$ interstimulus interval (fixation cross, “+”). In each fixation trial, a fixation cross was presented for the entire $3000 \mathrm{~ms}$. Participants practiced the task before the main experiment to ensure they understood the instructions and could perform accurately. The behavioral data were collected as part of an ongoing imaging study whose results will be reported elsewhere.

\section{DATA ANALYSIS}

Our analyses focused on determining whether post-error slowing and/or post-conflict adaptation differed among healthy youth and patients with pediatric OCD. Separate analyses were conducted on median reaction time (RT) and mean accuracy using PASW statistics 18. Significant main effects and/or interactions from the ANOVAs were followed up with planned contrasts. $t$-Tests and Pearson correlations were considered significant when their associated $p$-values were less than 0.05 (two-tailed).

\section{Post-error slowing}

To determine whether the magnitude of post-error slowing differed for the two groups, we analyzed current congruent and current incongruent trials separately. This ensured that the vastly different response times in these conditions would not confound the post-error analysis. Each analysis was conducted using a mixed ANOVA with previous trial type (correct, error) as a withinparticipants factor and group (patients, controls) as a betweenparticipants factor. Furthermore, each ANOVA included only those participants with at least five errorCorrect (i.e., correct trial after an error) trials. This resulted in 28 controls and 25 patients being included in the analysis of current incongruent trials, and 11 controls and 19 patients being included in the analysis of current congruent trials. Table 1 provides demographic and clinical measures for the sub-samples of patients and controls that were included in the analysis of post-error slowing in incongruent trials. Of note, these measures did not significantly differ from those in the participants who were excluded from the overall sample ( $p$-values $>0.10)$.

\section{Post-conflict adaptation}

To determine whether the magnitude of post-conflict adaptation differed for the two groups, we conducted separate three-way ANOVAs for RT and accuracy. Each ANOVA had three factors: previous trial type (i, incongruent and c, congruent) and current trial type (I, incongruent and $\mathrm{C}$, congruent) served as withinparticipants factors and group (patients, controls) served as a between-participants factor. Notably, our analyses of post-conflict adaptation excluded trials containing exact stimulus and response repetitions across consecutive trials because such repetitions can induce post-conflict adaptation effects via priming (Mayr et al., 2003) or, more generally, feature integration effects (Hommel et al., 2004).

Given that impaired conflict processing may characterize OCD (Chamberlain et al., 2006), we expected greater disruption of postconflict adaptation in patients when the current trial was incongruent (high-conflict) as compared to congruent (low-conflict). Thus, we further examined post-conflict adaptation separately for current congruent and current incongruent trials. Each of these two analyses was conducted using a mixed ANOVA with previous trial type (congruent, incongruent) as a within-participants factor and group (OCD, controls) as a between-participants factor.

\section{Correlations between trial-by-trial adjustments of cognitive control and clinical measures}

To test for relationships between trial-by-trial adjustments and symptom severity, we correlated post-error slowing and postconflict adaptation with current CYBOCs scores. In addition, we tested for effects of age and age by group interactions on posterror slowing and post-conflict adaptation. Finally, to explore the possible influence of medication on trial-by-trial adjustments of cognitive control, we compared post-error slowing and postconflict adaptation in medicated and unmedicated patients. In the post-error analyses, only participants with at least five errorCorrect trials were included.

\section{RESULTS}

\section{POST-ERROR SLOWING}

\section{Current congruent trials}

An ANOVA on median RT with previous trial type (correct, error) and group (patients, controls) as factors revealed no significant main effects or interactions (all $p>0.09$ ). In short, neither group exhibited post-error slowing in current congruent trials.

\section{Current incongruent trials}

An ANOVA on median RT with previous trial type (correct, error) and group (patients, controls) as factors revealed a significant 
interaction between previous trial type and group $[F(1,51)=5.24$, $p<0.05]$. While healthy youth slowed their responses after errors $[t(27)=2.17, p<0.05]$, patients showed nominally faster RTs following errors, relative to correct trials $[t(24)=-1.10, p>0.10$; Figure 2]. No other effects were significant.

\section{POST-CONFLICT ADAPTATION Median RT}

An ANOVA with previous trial type (congruent, incongruent), current trial type (congruent, incongruent), and group (patients, controls) revealed several significant main effects and interactions. First, there were main effects of previous trial type $[F(1,94)=10.73, p<0.001]$ and current trial type $[F(1,94)=721.09, p<0.001]$. Second, there were two-way interactions between previous trial type and current trial type $[F(1,94)=22.81, p<0.001]$ and between previous trial type and group $[F(1,94)=5.35, p<0.05]$. [Of note, there was no interaction between current trial type and group $(F<1, p=0.765)$. Thus, in this sample, the main effect of current trial type did not differ in patients and controls.] Third, there was a three-way interaction among all three factors $[F(1,94)=6.79, p<0.05]$. Visual inspection of the data suggested that post-conflict adaptation (i.e., faster responses in iI than in cI trials) was present in controls, but not in patients (Figure 3A).

This observation was supported by a series of follow-up analyses. First, in controls (Figure 3A, left), there was a significant interaction between previous trial type and current trial type $[F(1,47)=21.75, p<0.001]$, which was absent in patients $[F(1,47)=3.15, p>0.08$; Figure 3A, right $]$. Follow-up analyses revealed that both groups responded more quickly in $\mathrm{cC}$ trials than in $\mathrm{iC}$ trials [ 720 vs. $765 \mathrm{~ms}, F(1,94)=49.05, p<0.001]$ and that there was no group difference in the size of this effect $(F<1)$. However, while controls responded more quickly in iI than in cI trials [956 vs. $991 \mathrm{~ms}, t(47)=-4.51, p<0.001$; Figure 3A,

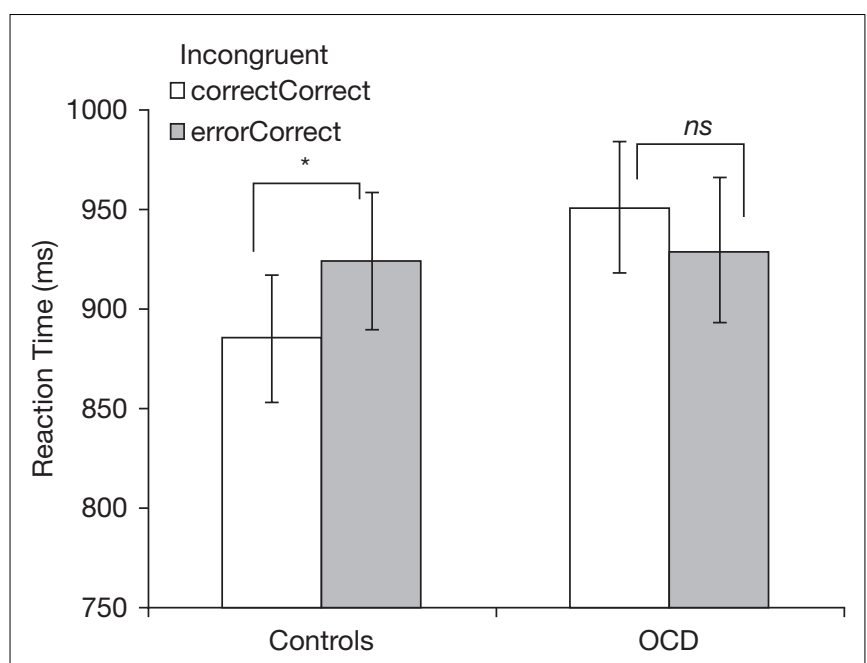

FIGURE 2 | Post-error slowing in incongruent trials for healthy controls (left) and patients with OCD (right). correctCorrect, correct incongruent trials following correct trials; errorCorrect, correct incongruent trials following error trials. ${ }^{*} p<0.05$; ns, not significant $(p>0.05)$. left], patients did not [1047 vs. $1026 \mathrm{~ms}, t(47)=1.71, p=0.09$; Figure 3, right], leading to an interaction between previous trial type and group for current incongruent trials $[F(1,94)=8.97$, $p<0.01]$. In sum, not all trial-by-trial adjustments were impaired in OCD patients; rather, patients showed a selective alteration of post-conflict adaptation.

\section{Mean accuracy}

There was a main effect of current trial type $[F(1,94)=162.11$, $p<0.001]$ and an interaction between current trial type and group $[F(1,94)=4.86, p<0.05$; Figure $3 \mathbf{B}]$. The main effect of current trial type was driven by lower accuracy in incongruent trials, relative to congruent trials, as previously reported in the MSIT (Bush et al., 2003; Fitzgerald et al., 2010). The interaction between current trial type and group was driven by a greater difference in accuracy between incongruent and congruent trials in patients, relative to controls. No other effects were significant (all $p>0.10)$.

\section{CORRELATIONS BETWEEN TRIAL-BY-TRIAL ADJUSTMENTS OF COGNITIVE CONTROL AND CLINICAL MEASURES}

There was no significant correlation between post-error slowing and current CYBOCS score in patients with pediatric OCD. However, there were significant correlations between post-conflict adaptation in incongruent trials and (a) current CYBOCS compulsion score $[r(45)=0.416, p<0.01$, Figure $4 \mathrm{~A}]$ as well as (b) total score [the sum of the compulsion and obsession scores, $r(45)=0.362, p<0.05]$. No correlation was found between postconflict adaptation in incongruent trials and obsession score alone $[r(45)=0.249, p>0.05]$.

To better characterize the correlation between post-conflict adaptation in incongruent trials and current CYBOCS compulsion score in OCD patients, we performed a two-way ANOVA on median RT for incongruent trials. The factors included previous trial type (iI, cI) and severity of compulsion (high, low). As the name of our second factor - severity of compulsion score implies, patients were categorized into two groups based on their current CYBOCS compulsion scale scores. One group was characterized as having high symptom severity (range of scores, $11-17 ; n=19$ ) while the other was characterized as having low symptom severity (range of scores, $0-8 ; n=19$ ). Nine participants with mid-range scores (i.e., 9 and 10) and one participant with missing values were excluded from this analysis.

The ANOVA revealed a main effect of previous trial type $\left[\mathrm{RT}_{\mathrm{iI}}>\mathrm{RT}_{\mathrm{cI}}, F(1,36)=5.06, p<0.05\right]$ and an interaction between previous trial type and group $[F(1,36)=12.90, p<0.001]$. Planned contrasts revealed that patients with low compulsion scores responded more slowly to iI than cI [1046 vs. $974 \mathrm{~ms}$, $t(18)=4.48, p<0.01$, two-tailed] while patients with high compulsion scores did not ( $\mathrm{RT}_{\mathrm{iI}}$ vs. $\mathrm{RT}_{\mathrm{cI}}, 1103$ vs. $1120 \mathrm{~ms}$; Figure 4B).

Although age was negatively correlated with median RT in both patients $(r=-0.690, p<0.001)$ and controls $(r=-0.720$, $p<0.001$ ), there was no group difference in the magnitude of this effect (i.e., no group $\times$ age interaction, $p>0.1$ ). Further, in both patients and controls, there was no correlation between age and post-error slowing, or between age and post-conflict adaptation. Finally, neither post-error slowing nor post-conflict adaptation 


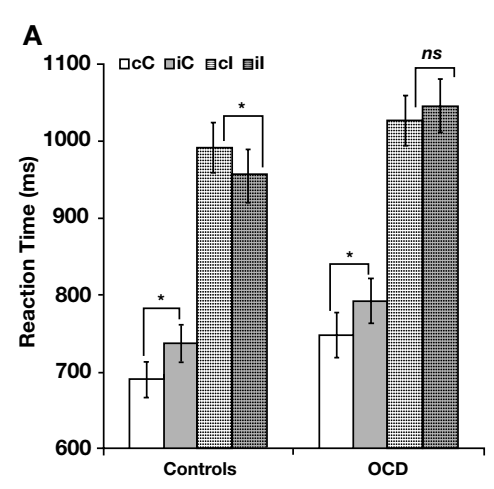

FIGURE 3 | Post-conflict adaptation effects in (A) reaction time and (B) accuracy. Each panel shows effects separately for healthy controls (left) and patients with $\mathrm{OCD}$ (right). $\mathrm{CC}$, congruent trials following congruent trials; iC,

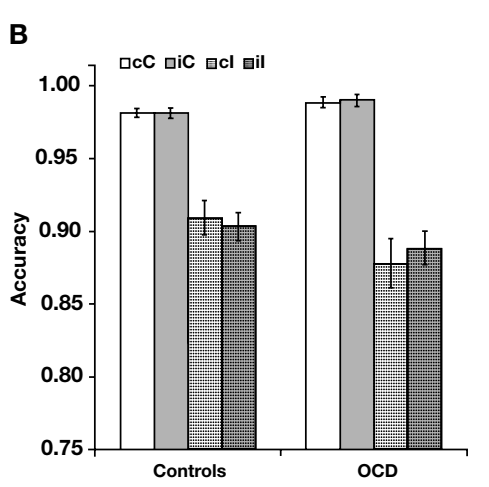

congruent trials following incongruent trials; $\mathrm{cl}$, incongruent trials following congruent trials; il, incongruent trials following incongruent trials. ${ }^{*} p<0.05$; ns, not significant $(p>0.05)$.

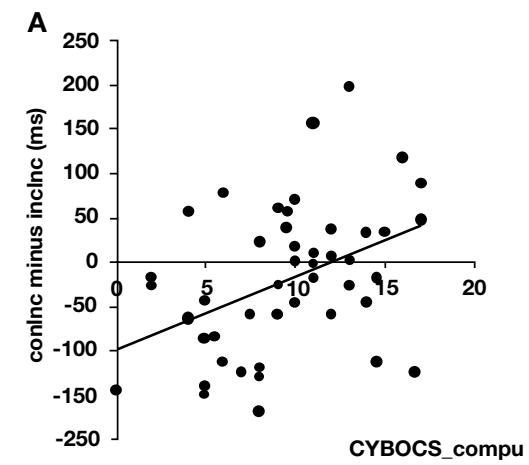

FIGURE 4 | Relationships between post-conflict adaptation in current incongruent trials and symptom severity in pediatric $O C D$. (A) Correlation between post-conflict adaptation in current incongruent trials and current CYBOCS compulsion score in OCD. (B) Post-conflict adaptation in current incongruent trials shown separately for healthy

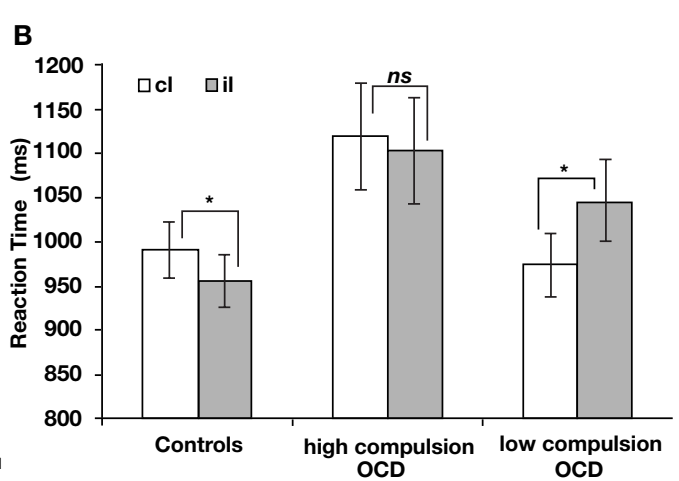

controls (left), patients with high present CYBOCS compulsion score (middle), and patients with low present CYBOCS compulsion score (right). cl, incongruent trials following congruent trials; il, incongruent trials following incongruent trials. ${ }^{*} p<0.05$; ns, not significant, $p>0.05$. differed between medicated and non-medicated patients (both $p$-values $>0.1$ ).

\section{DISCUSSION}

We investigated whether patients with pediatric OCD exhibit abnormal trial-by-trial adjustments of cognitive control, relative to healthy controls. In line with prior work, controls exhibited both post-error slowing and post-conflict adaptation in incongruent trials. Further, as predicted, patients did not exhibit either of these effects. These findings support our hypothesis that trial-by-trial adjustments of cognitive control are altered in pediatric OCD.

\section{POST-ERROR SLOWING}

Unlike healthy controls, patients with pediatric OCD did not slow their responses in incongruent trials following errors, relative to incongruent trials following correct responses. The absence of post-error slowing in pediatric OCD contrasts with prior work showing similar levels of post-error slowing in adult OCD (Endrass et al., 2008) and in college students with high (vs. low) OCD symptoms (Hajcak and Simons, 2002). Given these prior results, the present findings suggest that the absence of post-error slowing in incongruent trials may be specific to pediatric OCD. Given that children and adolescents find most tasks more difficult to perform than adults (Hogan et al., 2005; Friedman et al., 2009), we speculate that trial-by-trial adjustments of cognitive control may be reduced in pediatric OCD when attentional resources are depleted during difficult tasks (e.g., the MSIT). Future studies will be necessary to test this speculative hypothesis and to determine whether normal post-error slowing and conflict adaptation are observed in pediatric OCD during the performance of less demanding tasks.

Post-error slowing is thought to depend on intact frontostriatal circuitry (Ullsperger and von Cramon, 2006). Specifically, fMRI data from adults show that behavioral adjustments such as post-error slowing activate both the anterior cingulate cortex (ACC) and the lateral prefrontal cortex (LPFC, Kerns et al., 2004). The ACC is believed to monitor for high levels of response conflict (e.g., incongruent trials and errors) and, upon detecting such conflict, signal LPFC-mediated cognitive control mechanisms to improve subsequent performance (Kerns et al., 2004). Thus, the 
lack of post-error slowing in pediatric OCD may index a failure of ACC-LPFC interactions underlying cognitive control, consistent with recent work linking OCD to altered functioning of these regions (Menzies et al., 2008; Fitzgerald et al., 2010). Future studies might be conducted to directly investigate this hypothesis.

Future studies will also be needed to explore which psychological processes giving rise to post-error slowing are impaired in pediatric OCD. As mentioned earlier, post-error slowing may reflect the operation of performance monitoring processes that enable more careful, deliberative responding following an error (e.g., Botvinick et al., 2001). From this perspective, the lack of post-error slowing in pediatric OCD may reflect heightened performance monitoring in correctly performed trials (e.g., due to the intrusion of task-irrelevant thoughts) as well as in error trials, such that RT is longer in correctCorrect as well as in errorCorrect trials. Another possibility is that post-error slowing occurs because errors are rare, "oddball" events that draw attention away from the primary task (Notebaert et al., 2009). From this perspective, the lack of post-error slowing in pediatric OCD may reflect frequent task-irrelevant thoughts that draw attention away from the primary task in correct as well as in error trials. Clearly, additional work will be needed to distinguish these (and other) views.

Regardless of which view proves correct, the present findings are important because they indicate that patients with pediatric OCD fail to specifically recognize and/or react to errors. Such deficits may play a central role in OCD, wherein an inability to detect and/or react to errors of thinking (i.e., obsessions) may compromise patients' ability to resist compulsive behaviors and move on to more productive activities. Consistent with this possibility, a primary goal of cognitive behavioral therapy (CBT) is to teach OCD patients to identify obsessions as thinking errors that must be deliberately resisted. Also consistent, following successful CBT many patients become able to disengage from compulsive urges and orient their behavior toward achieving more productive goals.

\section{POST-CONFLICT ADAPTATION}

Patients with pediatric OCD also exhibited reduced post-conflict adaptation in current incongruent trials. Specifically, while healthy controls exhibited post-conflict adaptation in current incongruent trials (i.e., they responded more quickly in iI than cI trials), children with OCD did not, suggesting a failure to recruit control processes that enable behavioral adjustments. Interestingly, this deficit was specific to behavioral adjustments in current incongruent trials as no such abnormality was observed in current congruent trials wherein both groups responded more quickly in $\mathrm{cC}$ than in $\mathrm{iC}$ trials. This result shows that not all trial-by-trial adjustments of behavior are impaired in pediatric OCD. Rather, only those adjustments that must be implemented under highconflict conditions (i.e., during incongruent trials) are impaired. As we speculated earlier, problems with implementing behavioral adjustments under high-conflict conditions may relate to OCD patients' inability to resist compulsive behaviors (e.g., repeatedly washing one's hands).

\section{CORRELATIONS BETWEEN POST-CONFLICT ADAPTATION AND OCD SYMPTOMS}

At the level of individual patients, the magnitude of post-conflict adaptation varied with compulsive symptom scores. Patients with low scores exhibited a reverse post-conflict adaptation effect: they responded more slowly in iI than in cI trials. In contrast, patients with high scores showed no evidence of post-conflict adaptation. Generally speaking, these findings fit with our hypothesis that pediatric OCD is linked to deficits in cognitive control. However, the finding of a reverse post-conflict adaptation effect in patients with low scores is somewhat surprising. One possibility is that these patients experienced greater conflict in iI than in cI trials, possibly due to spillover from the prior incongruent trial. A second possibility is that even reverse post-conflict adaptation indexes the operation of cognitive control processes that function to optimize performance. Consistent with this second possibility, healthy adults sometimes exhibit post-conflict slowing (Verguts et al., 2011), rather than post-conflict speeding, and such slowing may serve to minimize errors in high-conflict incongruent trials (Ullsperger et al., 2005). We therefore speculate that the reverse post-conflict adaptation effect in patients with low symptom severity may reflect abnormally large post-conflict slowing, which leads to slower (not faster) responses in il trials than in cI trials. Furthermore, the complete lack of post-conflict adaptation in patients with high symptom severity may reflect exaggerated slowing following both congruent and incongruent trials, consistent with an abnormally high recruitment of performance monitoring processes after all trials. Consistent with this possibility, median RT was especially high in patients with high compulsive symptoms scores (Figure 4B, middle). In sum, the magnitude of post-conflict adaptation varied with compulsive symptom scores, but additional studies will be needed to explore various interpretations of these effects.

\section{LIMITATIONS}

While we excluded exact stimulus repetitions in our experiment, the nature of the MSIT prevented us from excluding partial stimulus repetitions. Partial stimulus repetitions occur when the stimulus that serves as the target or the distracter in one trial serves as either the target or the distracter in the next trial (e.g., the target in one trial becomes the distracter in the next trial). Notably, partial stimulus repetitions can influence the magnitude of conflict adaptation (Hommel et al., 2004; Egner, 2007). One might therefore wonder whether the group difference in conflict adaptation that we observed reflected a group difference in the effect of partial stimulus repetitions on performance, rather than a group difference in cognitive control.

Post hoc analyses argued against this possibility. In particular, although both patients and controls exhibited marginally faster response times in trials with partial stimulus repetitions than in trials without partial stimulus repetitions $[F(1,94)=3.67$, $p=0.058]$, there was no group difference in the size of this effect $[F(1,94)=1.254, p=0.266]$. These findings suggest that partial stimulus repetitions exerted equivalent effects in patients and controls, and that they did not account for the group difference in conflict adaptation that we observed. Nonetheless, future studies of conflict adaptation in OCD could improve upon the present work by using tasks in which partial stimulus repetitions can be excluded from all analyses.

Two other limitations of the present study relate to the absence of post-error slowing in congruent trials and to the relatively small 
sample size. With regard to the former, the absence of post-error slowing in congruent trials was observed in a relatively small subset of the participants and should therefore be interpreted with caution. With regard to the latter, the relatively small number of patients in this study (and their limited age range: 8-19 years) precludes a detailed investigation of whether and how trial-by-trial adjustments of cognitive control are altered in OCD across the entire lifespan. Future studies will be needed to address each of these limitations.

Our overall finding that OCD youth exhibit reduced post-error slowing contrasts with prior work indicating normal post-error slowing in adult OCD (Hajcak and Simons, 2002; Endrass et al., 2008). This discrepancy raises the possibility that our findings may only be relevant to understanding pediatric OCD. However, rather than being a limitation of our study, this finding suggests that control deficits associated with OCD may change over the lifespan. Longitudinal studies of OCD might therefore help to link the present findings with prior findings from the adult OCD literature (Hajcak and Simons, 2002; Endrass et al., 2008). Further, longitudinal studies of youth at risk for developing OCD (e.g.,

\section{REFERENCES}

American Psychiatric Association. (2000). Diagnostic and Statistical Manual of Mental Disorders, 4th Edn. Washington, DC: American Psychiatric Association.

Aycicegi, A., Dinn, W. M., Harris, C. L., and Erkmen, H. (2003). Neuropsychological function in obsessive-compulsive disorder: effects of comorbid conditions on task performance. Eur. Psychiatry 18, 241-248.

Bannon, S., Gonsalvez, C. J., Croft, R. J., and Boyce, P. M. (2002). Response inhibition deficits in obsessivecompulsive disorder. Psychol. Res. $110,165-174$.

Botvinick, M. M., Braver, T. S., Barch, D. M., Carter, C. S., and Cohen, J. D. (2001). Conflict monitoring and cognitive control. Psychol. Rev. 108, 624-652.

Bush, G., and Shin, L. M. (2006). The Multi-Source Interference Task: an fMRI task that reliably activates the cingulo-frontal-parietal cognitive/attention network. Nat. Protoc. 1, 308-313.

Bush, G., Shin, L. M., Holmes, J., Rosen, B. R., and Vogt, B. A. (2003). The Multi-Source Interference Task: validation study with fMRI in individual subjects. Mol. Psychiatry 8, 60-70.

Chamberlain, S. R., Fineberg, N. A., Blackwell, A. D., Robbins, T. W., and Sahakian, B. J. (2006). Motor inhibition and cognitive flexibility in obsessive-compulsive disorder and trichotillomania. Am. J. Psychiatry $163,1282-1284$.
Davies, P. L., Segalowitz, S. J., and Gavin, W. J. (2004). Development of response-monitoring ERPs in 7-25year-olds. Dev. Neuropsychol. 25, 355-376.

Egner, T. (2007). Congruency sequence effects and cognitive control. Cogn. Affect. Behav. Neurosci. 7, 380-390.

Egner, T., Etkin, A., Gale, S., and Hirsch, J. (2008). Dissociable neural systems resolve conflict from emotional versus non-emotional distracters. Cereb. Cortex 18, 1475-1484.

Egner, T., and Hirsch, J. (2005). Cognitive control mechanisms resolve conflict through cortical amplification of task-relevant information. Nat. Neurosci. 8, 1784-1790.

Endrass, T., Klawohn, J., Schuster, F., and Kathmann, N. (2008). Overactive performance monitoring in obsessive-compulsive disorder: ERP evidence from correct and erroneous reactions. Neuropsychologia 46, 1877-1887.

Enright, S. J., and Beech, A. R. (1993). Reduced cognitive inhibition in obsessive-compulsive disorder. $\mathrm{Br}$. J. Clin. Psychol. 32, 67-74.

Fitzgerald, K. D., Stern, E. R., Angstadt, M., Nicholson-Muth, K., Maynor, M. R., Welsh, R. C., Hanna, G. L., and Taylor, S. F. (2010). Altered function and connectivity of the medial frontal cortex in pediatric obsessivecompulsive disorder. Biol. Psychiatry 68, 1039-1047.

Fitzgerald, K. D., Welsh, R. C., Gehring, W. J., Abelson, J. L., Himle, J. A., Liberzon, I., and Taylor, S. F. (2005). Error-related hyperactivity

first degree relatives of OCD patients) might reveal whether alterations of cognitive control appear before or after the onset of OCD symptoms.

\section{CONCLUSION}

The present findings demonstrate that trial-by-trial adjustments of cognitive control are reduced in patients with pediatric OCD, relative to healthy youth. They also show that the magnitude of these reductions varies with symptom severity. Future studies of these effects may help to reveal early markers of the illness that are useful in both research and clinical settings.

\section{ACKNOWLEDGMENTS}

This work was supported by a Dana Foundation Award, a Todd Ouida Clinical Scholars Award, a National Alliance for Research in Schizophrenia and Affective Disorders (NARSAD) Young Investigator Award, and a National Institute of Mental Health (NIMH) Career Development Award (1K23-MH082176) to Kate Dimond Fitzgerald, and by NIMH R01-MH071821 to Stephan F. Taylor.

of the anterior cingulate cortex in obsessive-compulsive disorder. Biol. Psychiatry 57, 287-294.

Friedman, D., Nessler, D., Cycowicz, Y. M., and Horton, C. (2009). Development of and change in cognitive control: a comparison of children, young adults, and older adults. Cogn. Affect. Behav. Neurosci. 9, 91-102.

Goodman, W. K., Price, L. H., Rasmussen, S. A., Mazure, C., Fleischmann, R. L., Hill, C. L., Heninger, G. R., and Charney, D. S. (1989). The Yale-Brown Obsessive Compulsive Scale: I. Development, use, and reliability. Arch. Gen. Psychiatry 46 1006-1011.

Hajcak, G., and Simons, R. F. (2002). Error-related brain activity in obsessive compulsive undergraduates. Psychiatry Res. 110, 63-72.

Hogan, A. M., Vargha-Khadem, F., Kirkham, F. J., and Baldeweg, T. (2005). Maturation of action monitoring from adolescence to adulthood: an ERP study. Dev. Sci. 8, 525-534.

Hollingshead, A. B., and Redlich, F. C. (1958). Social class and mental illness. Am. J. Psychiatry 149, 1035-1044.

Hommel, B., Proctor, R. W., and Vu, K.P. L. (2004). A feature-integration account of sequential effects in the Simon task. Psychol. Res. 68, 1-17.

Kaufman, J., Birmaher, B., Brent, D., Rao, U., Flynn, C., Moreci, P., Williamson, D., and Ryan, N. (1997). Schedule for affective disorders and schizophrenia for school-age children - present and lifetime version (K-SADS-PL): initial reliability and validity data. $J$. Am Acad. Child Adolesc. Psychiatry 36, 980-988.

Kerns, J. G., Cohen, J. D., MacDonald, A. W. III, Cho, R. Y., Stenger, V. A., and Carter, C. S. (2004). Anterior cingulate conflict monitoring and adjustments in control. Science 303, 1023-1026.

Kovacs, M. (1992). Children's Depression Inventory (CDI) Manual. New York: Mental Health Systems.

Ladouceur, C. D., Dahl, R. E., and Carter, C. S. (2007). Development of action monitoring through adolescence into adulthood: ERP and source localization. Dev. Sci. 10, 874-891.

Laming, D. (1968). Information Theory of Choice-Reaction Times. New York: Academic Press.

March, J. S., Parker, J. D., Sullivan, K., Stallings, P., and Conners, C. K. (1997). The Multidimensional Anxiety Scale for Children (MASC): factor structure, reliability, and validity. J. Am. Acad. Child Adolesc. Psychiatry 36, 554-565.

Mayr, U., Awh, E., and Laurey, P. (2003). Post-conflict adaptation effects in the absence of executive control. Nat. Neurosci. 6, 450-452.

Meiran, N., Diamond, G. M., Toder, D., and Nemets, B. (2011). Cognitive rigidity in unipolar depression and obsessive compulsive disorder: examination of task switching, Stroop, working memory updating and post-conflict adaptation. Psychiatry Res. 185, 149-156. 
Menzies, L., Chamberlain, S. R., Laird, A. R., Thelen, S. M., Sahakian, B. J., and Bullmore, E. T. (2008). Integrating evidence from neuroimaging and neuropsychological studies of obsessive-compulsive disorder: the orbitofronto-striatal model revisited. Neurosci. Biobehav. Rev. 32, 525-549.

Norman, D. A., and Shallice, T. (1986). "Attention to action: willed and automatic control of behaviour," in Consciousness and Self Regulation, eds R. Davidson, G. Schwarz, and D. Shapiro (New York, NY: Plenum), 1-18.

Notebaert, W., Houtman, F., Van Opstal, F., Gevers, W., Fias, W., and Verguts, T. (2009). Post-error slowing: an orienting account. Cognition 111, 275-279.

Pauls, D. L., Alsobrook, J. P., Goodman, W., Rasmussen, S., and Leckman, J. F. (1995). A family study of obsessive-compulsive disorder. Am. J. Psychiatry 152, 76-84.
Rabbitt, P. M. (1966). Errors and error correction in choice response tasks. J. Exp. Psychol. 71, 264-272.

Rosenberg, D. R., Averbach, D. H., O'Hearn, K. M., Seymour, A. B., Birmaher, B., and Sweeney, J. A. (1997). Oculomotor response inhibition abnormalities in pediatric obsessive-compulsive disorder. Arch. Gen. Psychiatry 54, 831-838.

Santesso, D. L., Segalowitz, S. J., and Schmidt, L. A. (2006). Errorrelated electrocortical responses are enhanced in children with obsessivecompulsive behaviors. Dev. Neuropsychol. 29, 431-445.

Soref, A., Dar, R., Argov, G., and Meiran, N. (2008). Obsessivecompulsive tendencies are associated with a focused information processing strategy. Behav. Res. Ther. 45, 1295-1299.

Ullsperger, M., Bylsma, L. M., and Botvinick, M. M. (2005). The conflict-adaptation effect: it's not just priming. Cogn. Affect. Behav. Neurosci. 5, 467-472.

Ullsperger, M., and von Cramon, D. Y. (2006). The role of intact frontostriatal circuits in error processing. J. Cogn. Neurosci. 18, 651-664.

Verguts, T., Notebaert, W., Kunde, W., and Wühr, P. (2011). Post-conflict slowing: cognitive adaptation after conflict processing. Psychon. Bull. Rev. 18, 76-82.

Watkins, L. H., Sahakian, B. J., Robertson, M. M., Veale, D. M., Rogers, R. D., Pickard, K. M., Aitken, M. R., and Robbins, T. W. (2004). Executive function in Tourette's syndrome and obsessive-compulsive disorder. Psychol. Med. 34, $1-12$.

Conflict of Interest Statement: The authors declare that the research was conducted in the absence of any commercial or financial relationships that could be construed as a potential conflict of interest.

Received: 04 February 2012; paper pending published: 22 March 2012; accepted: 15 April 2012; published online: 11 May 2012.

Citation: Liu Y, Gehring WJ, Weissman DH, Taylor SF and Fitzgerald KD (2012) Trial-by-trial adjustments of cognitive control following errors and response conflict are altered in pediatric obsessive compulsive disorder. Front. Psychiatry 3:41. doi: 10.3389/fpsyt.2012.00041

This article was submitted to Frontiers in Child and Neurodevelopmental Psychiatry, a specialty of Frontiers in Psychiatry. Copyright (c) 2012 Liu, Gehring, Weissman, Taylor and Fitzgerald. This is an open-access article distributed under the terms of the Creative Commons Attribution Non Commercial License, which permits non-commercial use, distribution, and reproduction in other forums, provided the original authors and source are credited. 